\title{
Efficient Pricing of Derivatives on Assets with Discrete Dividends
}

\author{
M. H. VELLEKOOP* \& J. W. NIEUWENHUIS** \\ *Department of Applied Mathematics, University of Twente, The Netherlands, **Faculty of Economics, \\ Rijksuniversiteit Groningen, The Netherlands
}

(Received 14 July 2004; in revised form 16 November 2005)

\begin{abstract}
It is argued that due to inconsistencies in existing methods to approximate the prices of equity options on assets which pay out fixed cash dividends at future dates, a new approach to this problem may be useful. Logically consistent methods which are guaranteed to exclude arbitrage exist, but they are not very popular in practice due to their computational complexity. An algorithm is defined which is easy to understand, computationally efficient, and which guarantees to generate prices which exclude arbitrage possibilitites. It is shown that for the method to work a mild uniform convergence condition must be satisfied and this condition is indeed satisfied for standard European and American options. Numerical results testify to the accuracy and flexibility of the method.
\end{abstract}

KEY WorDS: Equity option, pricing dividends, numerical methods

\section{Introduction}

The incorporation of dividends in equity price models that are used to price derivatives on an underlying stock constitutes an important and non-trivial extension of such models. If a continuously paid dividend yield is used, or one is willing to specify the future dividends as a fixed percentage of the stock price at dividend dates, then the classicial option pricing model of Merton (1973), Black and Scholes (1973) can be used with only some minor modifications, but in reality option market makers prefer to specify dividends in terms of a fixed cash value instead of a percentage. This destroys the very feature which makes all option pricing computations so easy in the Black-Scholes model: the lognormal distribution of future stock prices. Standard approximation schemes such as the Cox, Ross and Rubinstein (1979) binomial tree methods can no longer be applied, or it becomes extremely inefficient from a computational point of view to do so.

As a result, certain approximations have been proposed in the literature to find option prices when the underlying asset pays cash dividends in the future. There are

Correspondence Address: M. H. Vellekoop, Department of Applied Mathematics, University of Twente, The Netherlands. Email: m.h.vellekoop@math.utwente.nl 
three basic modelling assumptions for such approximations that have been proposed in the literature, see for example the overview in Frishling (2002).

- Escrowed Model. Assume that the asset price minus the present value of all dividends to be paid until the maturity of the option follows a Geometric Brownian Motion.

- Forward Model. Assume that the asset price plus the forward value of all dividends (from past dividend dates to today) follows a Geometric Brownian Motion.

- Piecewise Lognormal Model. Assume that the stock price shows a jump downwards at dividend dates (equal to the cash dividend payments at those dates) and follows a Geometric Brownian Motion in between those dates.

Simple computations show that in the Escrowed and the Forward Model the prices for European options on dividend-paying assets reduce to the equivalent European prices on assets without dividend payments, but with an adjusted value of the current stock price or strike, respectively. Moreover, for American options it is quite easy to construct binomial trees which are still recombining and thus provide an efficient way to calculate derivative prices. This seems to be the reason why these approaches have been popular among practitioners. There are, however, serious modelling problems for these approaches. The Escrowed Model formulated above admits arbitrage opportunities, since one can easily show that for reasonable parameter values the American Call expiring just after the first dividend date can be cheaper than the American Call expiring just before the first dividend date (which is, therefore, equivalent to a European Call). The reason for this is obvious: different asset price process dynamics are assumed for the two products for the time up until the first dividend date. One can fix this by changing the definition to

- Modified Escrowed Model. Assume that the asset price minus the present value of all dividends to be paid in the future follows a Geometric Brownian Motion.

but this would mean that the prices of options will depend on the dividends which are being paid after the options have expired, which may be unsatisfactory as well, since this means that a trader would have to adjust the price of a two-year option once his view on the five-year dividend prediction changes.

It is therefore hardly surprising that the Piecewise Lognormal Model seems to be preferred from both a theoretical and a practical point of view, but it is often too inefficient for practical computation since discrete tree methods no longer recombine under this modelling assumption. Modifications of the Escrowed and Forward Models have therefore been proposed which bring the results closer to the Piecewise Lognormal Model:

- Adjusting the timing of dividends. Bos and Vandermark (2002) define a mixture of the Escrowed and Forward model, where part of the dividends is incorporated in a modified asset price, and part in a modified strike price. 
- Adjusting the volatility. Beneder and Vorst (2002), Bos et al. (2003) use a modified value for the volatility which incorporates the dividend payments and use this value in an Escrowed Model.

These modifications of the Escrowed and Forward Models usually give prices which are close to those generated by the Piecewise Lognormal Model. But they are still approximations which do not specify the stock price process underlying their models, but only adjust the parameters of the Black-Scholes formulas. This may explain why they give good results in the case of a single dividend, but their performance deteriorates when more than one cash dividend payment is considered.

In this paper we take a different approach. We take the Piecewise Lognormal Model as the given dynamics for the price process, and propose a method which overcomes the problem that standard CRR trees (which are used to approximate the prices) do not recombine after dividend dates. This has the important advantage that we do not need to define different asset dynamics for different products (due to differences in the number of dividends during the lifetime of products). Our method is based on interpolation steps within the tree which are easy to implement and take little time. Interpolation methods have been suggested before, for example in Wilmott et al. (1993), and Haug et al. (2003). Our method uses a different interpolation technique and does not suffer from the problem of possible negative risk-neutral probabilities in the first method (reported for example in Beneder and Vorst (2002)), and since it does not involve explicit integration methods such as in the second method, it will still work for American options as well as for European options.

Our method is logically consistent in the sense that we do not change the assumptions of the Piecewise Lognormal Model, and that it is guaranteed to be free of arbitrage, while events after expiry of a derivative do not influence the derivative price. Since it is simply a minor adjustment which keeps the tree recombination properties, it can be used equally well for American and European option pricing, and it will turn out to be rather trivial to make the cash dividend payments depend on the stock price just before the dividend date. This will also allow us to make sure that we cannot end up with negative stock prices after a dividend date as a result of low stock prices just before the stock goes ex-dividend. Stock-price dependent dividend payments were introduced in Haug et al. (2003) and we refer to the excellent analysis given in that paper for arguments in favour of this flexibility. We also note that it would be very easy to modify our method in such a way that one would define a new value for the volatility in the tree directly after each dividend date.

In the next section we describe our approach in more detail. The third section contains the conditions to make the method work, and gives a formal proof of convergence to the correct prices. This is the main result of the paper, since the proof that the approximations convergence to the right price under all cicumstances is the most important (and the hardest) part. In fact, our research has been motivated by questions from market makers who asked for a method for which convergence can be guaranteed, even for American options with multiple dividends. We prove our convergence results for the specific case of a binomial model, although one can use such interpolation methods in finite-difference schemes as well of course. The fourth 
section contains numerical results which show the performance of the method in practice, and in the last section we formulate conclusions and directions for further research.

\section{The Method}

As stated in the introduction, the way to handle dividends which seems to be preferred in general, is to define the following risk-neutral stochastic dynamics for the price process of the underlying (the Piecewise Lognormal Model)

$$
\begin{gathered}
\frac{\mathrm{d} S_{t}}{S_{t-}}=r \mathrm{~d} t+\sigma \mathrm{d} W_{t}+\frac{\mathrm{d} A_{t}}{S_{t-}} \\
\frac{\mathrm{d} B_{t}}{B_{t}}=r \mathrm{~d} t
\end{gathered}
$$

where $r>0$ and $\sigma>0$ are known constants, and

$$
A_{t}=-\sum_{i=1}^{n_{D}} D_{i}\left(S_{t_{i-}}\right) 1_{\left\{t \geq t_{i}\right\}}
$$

where $0<t_{1}<t_{2} \ldots<t_{n_{D}}$ are the $n_{D}$ times when dividends are being paid, while for all $i=1 \ldots n_{D}$, the $D_{i}: \mathbb{R}^{+} \rightarrow \mathbb{R}^{+}$are continuous and monotone functions which represent the amount of dividend (in cash) which is being paid at time $t_{i}$, and they are thus assumed to satisfy the constraint that $D_{i}(s) \leqslant s$, for all $s \geqslant 0$ and all $i \in\left\{1,2, \ldots, n_{D}\right\}$. The stochastic process $\left\{W_{t}, t \geqslant 0\right\}$ is a standard Brownian motion on a filtered probability space $\left(\Omega, \mathcal{F},\left(\mathcal{F}_{t}\right)_{t \geq 0}, \mathbb{Q}\right)$ with a risk-neutral probability measure $\mathbb{Q}$ and a filtration $\left(\mathcal{F}_{t}\right)_{t \geq 0}$ which satisfies the usual assumptions. The processes $S$ and $B$ are thus completely specified under $\mathbb{Q}$ once their initial conditions $S_{0}$ and $B_{0}$ have been specified.

We denote for all bounded Borel-sets $E \subset \mathbb{R}$ by $\mathcal{T}_{E}$ the class of stopping times (with respect to the filtration $\left.\left(\mathcal{F}_{t}\right)_{t \geq 0}\right)$ which take values in $E$, i.e.

$$
\tau \in \mathcal{T}_{E} \Rightarrow \mathbb{Q}(\tau \in E)=1, \quad\{\varpi: \tau(\varpi) \leq t\} \in \mathcal{F}_{t} \quad(\forall t \in E)
$$

For Lipschitz, polynomially bounded functions $\Phi: \mathbb{R}^{+} \rightarrow \mathbb{R}$ we can now define for all $s \geqslant 0$ the European and American Option price functions associated with the contingent claim $\Phi$ and maturity $T>0$ :

$$
\begin{aligned}
& V_{\mathrm{Eur}}(s)=B_{0} \mathbb{E}^{\mathbb{Q}}\left[\frac{\Phi\left(S_{T}\right)}{B_{T}} \mid S_{0}=s\right] \\
& V_{\mathrm{Am}}(s)=B_{0} \sup _{\tau \in \mathcal{T}_{[0, T]}} \mathbb{E}^{\mathbb{Q}}\left[\frac{\Phi\left(S_{\tau}\right)}{B_{\tau}} \mid S_{0}=s\right]
\end{aligned}
$$

When $n_{D}=0$ (no dividends) good and fast approximations for these functions can be found, for example using the binomial trees pioneered by Cox, Ross and Rubinstein (1979). We define a more general discrete approximation process. Once the initial value $S_{0}$ and the time of maturity $T>0$ have been fixed, we define for all 
$n \in \mathbb{N}^{+}$a time grid

$$
\Delta_{n}=T / n, \quad \mathbb{T}^{n}=\left\{k \Delta_{n}, k=0 \ldots n\right\}
$$

and a sequence of approximating discrete time processes $\left(S^{n}, B^{n}\right)=\left(S_{i \Delta_{n}}^{n}, B_{i \Delta_{n}}^{n}\right)_{i=0 . . n}$ where we take for every $n$ an appropriate filtered probability space with risk-neutral measure $\mathbb{Q}^{n}$ and

$$
\begin{aligned}
S_{(i+1) \Delta_{n}}^{n} & =S_{i \Delta_{n}}^{n} J_{i+1}^{n}, \quad S_{0}^{n}=S_{0} \\
B_{i \Delta_{n}}^{n} & =e^{-r \cdot i \Delta_{n}}
\end{aligned}
$$

with $J_{i}^{n}$ an $\mathcal{F}_{i \Delta_{n}}$-measurable stochastic variable. The approximation to the European and American Option price functions is

$$
\begin{aligned}
& v_{n}^{\text {Eur }}(s)=B_{0} \mathbb{E}^{\mathbb{Q}^{n}}\left[\Phi\left(S_{T}^{n}\right) / B_{T}^{n} \mid S_{0}=s\right] \\
& v_{n}^{\operatorname{Am}}(s)=B_{0} \sup _{\tau \in \mathcal{T}} \mathbb{\mathcal { T }}_{\left\{0, \Delta_{n}, 2 \Delta_{n}, \ldots, T\right\}} \mathbb{E}^{\mathbb{Q}^{n}}\left[\Phi\left(S_{\tau}^{n}\right) / B_{\tau}^{n} \mid S_{0}=s\right]
\end{aligned}
$$

Many possible choices for the distribution of $J_{i}^{n}$ under the corresponding discretetime martingale measure $\mathbb{Q}^{n}$ exist. One can take a binomial or trinomial model or any other stochastic processes which converges (weakly) to the Black-Scholes model. The most famous of these methods is the CRR-method of binomial trees, for which we have the following result.

Lemma 1. Assume $n_{D}=0$ (i.e. there are no dividends) and that the payoff function is that of a call or put, i.e. $\Phi(s)=(s-K)^{+}$or $\Phi(s)=(K-s)^{+}$for some $K>0$. For the model defined above with

$$
\mathbb{Q}^{n}\left(J_{i+1}^{n}=u_{n}\right)=1-\mathbb{Q}^{n}\left(J_{i+1}^{n}=d_{n}\right)=\frac{e^{r \Delta_{n}}-d_{n}}{u_{n}-d_{n}}, \quad u_{n}=\frac{1}{d_{n}}=e^{\sigma \sqrt{\Delta_{n}}}
$$

we then have, for all $s \geqslant 0$,

$$
\begin{aligned}
& \lim _{n \rightarrow \infty} v_{n}^{\mathrm{Eur}}(s)=V^{\mathrm{Eur}}(s) \\
& \lim _{n \rightarrow \infty} v_{n}^{\mathrm{Am}}(s)=V^{\mathrm{Am}}(s)
\end{aligned}
$$

and this convergence is uniform on closed bounded sets.

Proof. See for example Amin and Khanna (1994), Lamberton (1998) and Leisen (1998) for detailed proofs.

In principle, extension of these methods to the case where cash dividends are being paid is trivial. However, a direct approach often leads to non-recombining trees. For example, in the CRR-method of the previous lemma, we have that if a dividend $D$ has been paid at timestep $m$, we have

$$
\left(S\left(u_{n}\right)^{i}\left(d_{n}\right)^{m-i}-D\right) d_{n} \neq\left(S\left(u_{n}\right)^{i-1}\left(d_{n}\right)^{m-i+1}-D\right) u_{n}
$$

so one would have to build a new tree from every node after each dividend date 
which makes efficient computation for realistic problems impossible. We therefore propose to make the tree recombining again by using an interpolation technique after each dividend date. As an illustration, assume we have only one dividend date $t_{D}$ (i.e. $n_{D}=1$ here), and define the set of nodes at the dividend date

$$
A^{n}=\left\{s: \mathbb{Q}^{n}\left(S_{m(n) \Delta_{n}}^{n}=s\right)>0\right\}
$$

with $m(n) \in \mathbb{N}$ defined by the condition that

$$
m(n) \Delta_{n} \leq t_{D}<(m(n)+1) \Delta_{n}
$$

As before, we also have the set of nodes at maturity

$$
\bar{A}^{n}=\left\{s: \mathbb{Q}^{n}\left(S_{T}^{n}=s\right)>0\right\} .
$$

We then suggest the following procedure.

\section{Implementation}

- Build a binomial tree with $n$ time steps as usual. At the end of the tree we can now calculate the value of $\Phi(s)$ for all $s \in \bar{A}^{n}$. We now work backwards through the binomial tree as usual until we arrive at a dividend date (i.e. to timestep $m(n)$ ). The result of having worked backwards through the tree is the set of (approximate) values $f^{n}(s)$ for the option contract in all the points $s$ of the binomial tree at timestep $m(n)$, i.e. for all $s \in A^{n}$. These values $f^{n}(s)$ approximate the option values at a time just after the dividend has been paid, given that the stock price at that time equals $s$.

- To be able to continue backwards through the tree we now have to find the option values just before the dividend is paid. Since the stock price jumps down with an amount $D\left(S_{t-}\right)$ when it goes ex-dividend, the option value for a stock price $s$ just before the dividend date equals the option value for a stock price $s-D(s)$ just after the dividend date. We thus need the option values $f_{n}$ in all the points $s-D(s)$ where $s \in A^{n}$, but we have only calculated the values of $f^{n}(s)$ where $s \in A^{n}$. We therefore define a function which approximates the function $f^{n}$ on the whole of $\mathbb{R}^{+}$, based on the values of $f^{n}$ on $A^{n}$. This interpolating function is denoted by $\mathcal{B}_{f^{n}}^{n}$, and we can then calculate the values $\mathcal{B}_{f^{n}}^{n}(s-D(s))$ as approximations for the values of $f^{n}(s-D(s))$ with $s \in A^{n}$, which we need to continue the binomial method in the point $s$.

- After that we work further backwards through the tree, until we reach time zero, and the option value has been found.

The extension of this method to more than one dividend is obvious. Also note that it is not essential to use a binomial tree, but in the numerical examples given later, we will always use binomial trees.

It is clear that the convergence of this approximating procedure to the correct value when the number of time steps $n$ goes to infinity, will depend on the approximation procedure $\mathcal{B}$ that we use in the algorithm. Even when the quality of the approximation becomes better as $n$ increases, we cannot immediately conclude 
that our option price approximations will converge to the correct values when $n$ goes to infinity, because (1) our interpolation is based on values which still contain an error (i.e. the values $\left\{f^{n}(s), s \in A^{n}\right\}$ that we use to interpolate are only an approximation of the true option values in those points); and (2) the points $A^{n}$ in between which we approximate change as a function of $n$, and fill out the whole of $\mathbb{R}^{+}$only in the limit.

Because of this, we will need to pose explicit conditions on our interpolation procedure in the next section, to be able to prove there that convergence does indeed take place under the restrictions posed. This will be the subject of the next section.

\section{Formal Proof of Convergence}

We define the following option price functions that we would like to approximate

$$
\begin{aligned}
& V^{\operatorname{Eur}}(s)=B_{0} \mathbb{E}^{\mathbb{Q}}\left[\frac{\Phi\left(S_{T}\right)}{B_{T}} \mid S_{0}=s\right] \\
& V^{\operatorname{Am}}(s)=B_{0} \sup _{\tau \in \mathcal{T}_{[0, T]}} \mathbb{E}^{\mathbb{Q}}\left[\frac{\Phi\left(S_{\tau}\right)}{B_{\tau}} \mid S_{0}=s\right]
\end{aligned}
$$

with the dynamics of $S_{t}$ under $\mathbb{Q}$ defined as in (1), but with only one dividend, i.e. $n_{D}=1$ (the more general case can be handled similarly). We first define two functions which represent the pricing functions directly after the dividend has been paid

$$
\begin{aligned}
& f^{\mathrm{Eur}}(s)=B_{t_{D}} \mathbf{E}^{\mathbb{Q}}\left[\frac{\Phi\left(S_{T}\right)}{B_{T}} \mid S_{t_{D}}=s\right] \\
& f^{\mathrm{Am}}(s)=B_{t_{D}} \sup _{\tau \in \mathcal{T}_{] t_{D}, T\right]}} \mathbb{E}^{\mathbb{Q}}\left[\frac{\Phi\left(S_{\tau}\right)}{B_{\tau}} \mid S_{t_{D}}=s\right]
\end{aligned}
$$

Lemma 2. Under the assumptions stated above we have

$$
\begin{aligned}
& V^{\mathrm{Eur}}(s)=B_{0} \mathbb{E}^{\mathbb{Q}}\left[\frac{f^{\mathrm{Eur}}\left(S_{t_{D}-}-D\left(S_{t_{D}-}\right)\right)}{B_{t_{D}}} \mid S_{0}=s\right] \\
& V^{\mathrm{Am}}(s)=B_{0} \sup _{\tau \in \mathcal{T}} \mathbb{E}_{\left[0, t_{D}\right]}^{\mathbb{Q}}\left[\frac{\Phi\left(S_{\tau}\right)}{B_{\tau}} 1_{\left\{\tau<t_{D}\right\}}+1_{\left\{\tau=t_{D}\right\}} \frac{f^{\mathrm{Am}}\left(S_{t_{D}-}-D\left(S_{t_{D}-}\right)\right)}{B_{t_{D}}} \mid S_{0}=s\right]
\end{aligned}
$$

Proof. The European case is trivial, since

$$
\begin{aligned}
\mathbb{E}^{\mathbb{Q}}\left[\frac{\Phi\left(S_{T}\right)}{B_{T}} \mid S_{0}=s\right] & =\mathbb{E}^{\mathbb{Q}}\left[\mathbb{E}^{\mathbb{Q}}\left[\frac{\Phi\left(S_{T}\right)}{B_{T}} \mid S_{t_{D}}\right] \mid S_{0}=s\right] \\
& =\mathbb{E}^{\mathbb{Q}}\left[\frac{f^{\text {Eur }}\left(S_{t_{D}}\right)}{B_{t_{D}}} \mid S_{0}=s\right] \\
& =\mathbb{E}^{\mathbb{Q}}\left[\frac{f^{\text {Eur }}\left(S_{t_{D}-}-D\left(S_{t_{D}-}\right)\right)}{B_{t_{D}}} \mid S_{0}=s\right]
\end{aligned}
$$


For the American case, we note that

$$
\begin{aligned}
& V^{\operatorname{Am}}(s) / B_{0} \\
& =\sup _{\tau \in \mathcal{T}_{[0, T]}} \mathbb{E}^{\mathbb{Q}}\left[\frac{\Phi\left(S_{\tau}\right)}{B_{\tau}} 1_{\left\{\tau<t_{D}\right\}}+\frac{\Phi\left(S_{\tau}\right)}{B_{\tau}} 1_{\left\{\tau \geq t_{D}\right\}} \mid S_{0}=s\right] \\
& =\sup _{\tau \in \mathcal{T}_{[0, T]}} \mathbb{E}^{\mathbb{Q}}\left[\frac{\Phi\left(S_{\tau}\right)}{B_{\tau}} 1_{\left\{\tau<t_{D}\right\}}+1_{\left\{\tau \geq t_{D}\right\}} \mathbb{E}^{\mathbb{Q}}\left[\frac{\Phi\left(S_{\tau}\right)}{B_{\tau}} \mid \mathcal{F}_{t_{D}}\right] \mid S_{0}=s\right] \\
& \leq \sup _{\tau \in \mathcal{T}_{[0, T]}} \mathbb{E}^{\mathbb{Q}}\left[\frac{\Phi\left(S_{\tau}\right)}{B_{\tau}} 1_{\left\{\tau<t_{D}\right\}}+1_{\left\{\tau \geq t_{D}\right\}} \sup _{\zeta \in \mathcal{T}_{\left[t_{D}, T\right]}} \mathbb{E}^{\mathbb{Q}}\left[\frac{\Phi\left(S_{\zeta}\right)}{B_{\zeta}} \mid S_{t_{D}}\right] \mid S_{0}=s\right]
\end{aligned}
$$

but the last inequality is in fact an equality. To see this, assume that there exist a $\tau^{*} \in \mathcal{T}_{[0, T]}$ and $\zeta^{*} \in \mathcal{T}_{\left[t_{D}, T\right]}$ such that

$$
\begin{aligned}
& \mathbb{E}^{\mathbb{Q}}\left[\frac{\Phi\left(S_{\tau^{*}}\right)}{B_{\tau^{*}}} 1_{\left\{\tau^{*}<t_{D}\right\}}+1_{\left\{\tau^{*} \geq t_{D}\right\}} \mathbb{E}^{\mathbb{Q}}\left[\frac{\Phi\left(S_{\zeta^{*}}\right)}{B_{\zeta^{*}}} \mid S_{t_{D}}\right] \mid S_{0}=s\right] \\
& >\sup _{\tau \in \mathcal{T}_{[0, T]}} \mathbb{E}^{\mathbb{Q}}\left[\frac{\Phi\left(S_{\tau}\right)}{B_{\tau}} 1_{\left\{\tau<t_{D}\right\}}+1_{\left\{\tau \geq t_{D}\right\}} \mathbb{E}^{\mathbb{Q}}\left[\frac{\Phi\left(S_{\tau}\right)}{B_{\tau}} \mid \mathcal{F}_{t_{D}}\right] \mid S_{0}=s\right]
\end{aligned}
$$

then we can define the random variable

$$
\widetilde{\tau}=\tau^{*} 1_{\left\{\tau^{*}<t_{D}\right\}}+\zeta^{*} 1_{\left\{\tau^{*} \geq t_{D}\right\}}
$$

for which one can easily check that it is a stopping time in $\mathcal{T}_{[0, T]}$ as well, so the righthand side of (3) must be equal to or larger than

$$
\begin{aligned}
& \mathbb{E}^{\mathbb{Q}}\left[\frac{\Phi\left(S_{\tilde{\tau})}\right.}{B_{\tilde{\tau}}} 1_{\left\{\tilde{\tau}<t_{D}\right\}}+1_{\left\{\tilde{\tau} \geq t_{D}\right\}} \mathbb{E}^{\mathbb{Q}}\left[\frac{\Phi\left(S_{\tilde{\tau})}\right)}{B_{\tilde{\tau}}} \mid \mathcal{F}_{t_{D}}\right] \mid S_{0}=s\right] \\
& =\mathbb{E}^{\mathbb{Q}}\left[\frac{\Phi\left(S_{\tau^{*}}\right)}{B_{\tau^{*}}} 1_{\left\{\tau^{*}<t_{D}\right\}}+1_{\left\{\tau^{*} \geq t_{D}\right\}} \mathbb{E}^{\mathbb{Q}}\left[\frac{\Phi\left(S_{\zeta^{*}}\right)}{B_{\zeta^{*}}} \mid S_{t_{D}}\right] \mid S_{0}=s\right]
\end{aligned}
$$

which is the left-hand side of (3), which would be a contradiction. We thus conclude that

$$
\begin{aligned}
& V^{\operatorname{Am}}(s) / B_{0} \\
& =\sup _{\tau \in \mathcal{T}} \mathbb{E}_{[0, T]}^{\mathbb{Q}}\left[\frac{\Phi\left(S_{\tau}\right)}{B_{\tau}} 1_{\left\{\tau<t_{D}\right\}}+1_{\left\{\tau \geq t_{D}\right\}} \sup _{\zeta \in \mathcal{T}_{\left[t_{D}, T\right]}} \mathbb{E}^{\mathbb{Q}}\left[\frac{\Phi\left(S_{\zeta}\right)}{B_{\zeta}} \mid S_{t_{D}}\right] \mid S_{0}=s\right]
\end{aligned}
$$

and using the fact that if $\tau$ is a stopping time, so is $\tau \wedge t_{D}$, we find 


$$
\begin{aligned}
& V^{\mathrm{Am}}(s) / B_{0} \\
& =\sup _{\tau \in \mathcal{T}} \mathbb{E}_{\left[0, t_{D}\right]}^{\mathbb{Q}}\left[\frac{\Phi\left(S_{\tau}\right)}{B_{\tau}} 1_{\left\{\tau<t_{D}\right\}}+1_{\left\{\tau=t_{D}\right\}} \sup _{\zeta \in \mathcal{T}_{\left[t_{D}, T\right]}} \mathbb{E}^{\mathbb{Q}}\left[\frac{\Phi\left(S_{\zeta}\right)}{B_{\zeta}} \mid S_{t_{D}}\right] \mid S_{0}=s\right] \\
& =\sup _{\tau \in \mathcal{T}} \mathbb{E}_{\left[0, t_{D}\right]}^{\mathbb{Q}}\left[\frac{\Phi\left(S_{\tau}\right)}{B_{\tau}} 1_{\left\{\tau<t_{D}\right\}}+\frac{f^{\mathrm{Am}}\left(S_{t_{D}}\right)}{B_{t_{D}}} 1_{\left\{\tau=t_{D}\right\}} \mid S_{0}=s\right] \\
& =\sup _{\tau \in \mathcal{T}} \mathbb{E}_{\left[0, t_{D}\right]}^{\mathbb{Q}}\left[\frac{\Phi\left(S_{\tau}\right)}{B_{\tau}} 1_{\left\{\tau<t_{D}\right\}}+\frac{f^{\mathrm{Am}}\left(S_{t_{D}-}-D\left(S_{t_{D}-}\right)\right)}{B_{t_{D}}} 1_{\left\{\tau=t_{D}\right\}} \mid S_{0}=s\right]
\end{aligned}
$$

and this proves the result.

For all functions $g: A^{n} \rightarrow \mathbb{R}$ we need to define an approximating function which defines values for points in the set $\bar{A}^{n}=\left\{a-D(a), a \in A^{n}\right\}$, and we will denote these values by $\mathcal{B}_{g}^{n}(s)$ for $s \in \bar{A}^{n}$. We will do this in a relatively simple way, by using linear interpolation on the values of $\bar{A}^{n}$ augmented by the value in $S=0$, which will, for example, be zero for a call and equal to the strike $K$ for theput, and will be a known constant for all other types of options as well. This guarantees that we can indeed use interpolation for all values in $\bar{A}^{n}$ and do not need to extrapolate.

In contrast to the case where we had no dividends, we now use the following approximations, as suggested by the previous lemma

$$
\begin{aligned}
& V_{n}^{\text {Eur }}(s)=B_{0}^{n} \mathbb{E}^{\mathbb{Q}^{n}}\left[\frac{\mathcal{B}_{f_{n}^{\text {Eur }}}^{n}\left(S_{t_{D}^{n}-}^{n}-D\left(S_{t_{D}^{n}-}^{n}\right)\right)}{B_{t_{D}}^{n}} \mid S_{0}=s\right] \\
& V_{n}^{\operatorname{Am}}(s)=B_{0}^{n} \sup _{\tau \in \mathcal{T}} \mathbb{E}_{\left\{0, \Delta_{n}, \ldots, t_{D}^{n}\right\}}^{\mathbb{Q}^{n}}\left[\frac{\Phi\left(S_{\tau}^{n}\right)}{B_{\tau}} 1_{\left\{\tau<t_{D}\right\}}+1_{\left\{\tau=t_{D}\right\}} \frac{\mathcal{B}_{f_{n}^{\text {Am }}}^{n}\left(S_{t_{D}^{n}-}^{n}-D\left(S_{t_{D}^{n}-}^{n}\right)\right)}{B_{t_{D}}} \mid S_{0}=s\right]
\end{aligned}
$$

with the functions $f_{n}^{\text {Eur }}, f_{n}^{\text {Am }}$ defined by

$$
\begin{aligned}
& f_{n}^{\text {Eur }}(s)=B_{t_{D}^{n}}^{n} \mathbb{E}^{\mathbb{Q}^{n}}\left[\Phi\left(S_{T}^{n}\right) / B_{T}^{n} \mid S_{t_{D}^{n}}=s\right] \\
& f_{n}^{\operatorname{Am}}(s)=B_{t_{D}^{n}}^{n} \sup _{\tau \in \mathcal{T}} \mathbb{E}_{\left\{t_{D}^{n}, \ldots, T\right\}}^{\mathbb{Q}^{n}}\left[\Phi\left(S_{\tau}^{n}\right) / B_{\tau}^{n} \mid S_{t_{D}^{n}}=s\right]
\end{aligned}
$$

and

$$
t_{D}^{n}=m(n) \Delta_{n}, \quad t_{D-}^{n}=(m(n)-1) \Delta_{n}
$$

We will use the following assumptions:

- (A1) For all $n$, the stochastic process $S_{i \Delta_{n}}^{n} / B_{i \Delta_{n}}^{n}$ is Markov, it is a $\mathbb{Q}^{n}$ martingale, i.e. for all $i \in\{0,1, \ldots, n\}$ 


$$
\mathbb{E}^{\mathbb{Q}^{n}}\left[\frac{S_{(i+1) \Delta_{n}}^{n}}{B_{(i+1) \Delta_{n}}^{n}} \mid \frac{S_{i \Delta_{n}}^{n}}{B_{i \Delta_{n}}^{n}}\right]=\frac{S_{i \Delta_{n}}^{n}}{B_{i \Delta_{n}}^{n}}
$$

and the sequence of stochastic variables $\left\{S_{t_{D-}^{n}}^{n}, n \in \mathbb{N}^{+}\right\}$is uniformly integrable under $\mathbb{Q}^{n}$, i.e.

$$
\lim _{M \rightarrow \infty} \sup _{n} \mathbb{E}^{\mathbb{Q}^{n}}\left[S_{t_{D-}^{n}}^{n} 1_{\left\{S_{t^{n}}^{n} \geq M\right\}}\right]=0
$$

- (A2) The function $\Phi: \mathbb{R}^{+} \rightarrow \mathbb{R}$ satisfies a uniform Lipschitz condition, i.e.

$$
\left(\exists h_{\Phi}>0\right)\left(\forall x, y \in \mathbb{R}^{+}\right) \quad|\Phi(x)-\Phi(\mathrm{y})| \leq h_{\Phi}|x-y|
$$

and its absolute value is bounded by a polynomial.

- (A3) The approximating sequence of discrete time option prices is such that in the absence of dividends (i.e. when the function $D$ equals zero on its entire domain) we have uniform convergence (on closed, bounded sets) to the correct values, i.e. the conclusion of Lemma 1 holds for all payoff functions $\Phi$ satisfying condition (A2).

It is well known that condition (A3) is satisfied for standard calls and puts in the CRR-model. The hard part to prove is uniform convergence for the American Put, see for example the paper by Lamberton (1998) for a proof. The European case (which includes the American Call if there are no dividends) is relatively easy. Condition (A1) is certainly satisfied in the CRR-model, since the process $S^{n}$ converges weakly to the continuous process $S$ in that case.

We will first prove one more lemma that will be needed in the proof for our main theorem.

Lemma 3. Under conditions (A1) and (A2) we have that $f^{\mathrm{Am}}$ and $f^{\text {Eur }}$ are uniformly Lipschitz, and so are the functions $f_{n}^{\mathrm{Am}}$ and $f_{n}^{\text {Eur }}$ for all possible $n \in \mathbb{N}^{+}$.

Proof. As an example, we prove the result for the functions $f_{n}^{\text {Am }}$, all other cases can be handled similarly. Define the stochastic variable $G\left(s_{1}, s_{2}, t\right)$ for $s_{1}, s_{2} \in \mathbb{R}^{+}$and $k \in\{m(n), \ldots, n\}$ as

$$
G\left(s_{1}, s_{2}, k\right)=B_{t_{D}^{n}}^{n} \frac{\Phi\left(s_{1} \frac{S_{k \Delta_{n}}^{n}}{S_{t_{D}^{n}}^{n}}\right)-\Phi\left(s_{2} \frac{S_{k \Delta_{n}}^{n}}{S_{t_{D}^{n}}^{n}}\right)}{B_{k \Delta_{n}}^{n}}
$$

then for all stopping time $\zeta \in \mathcal{T}_{\left\{0, \Delta_{n}, . ., T\right\}}$, by the optional stopping theorem, equation (4) 


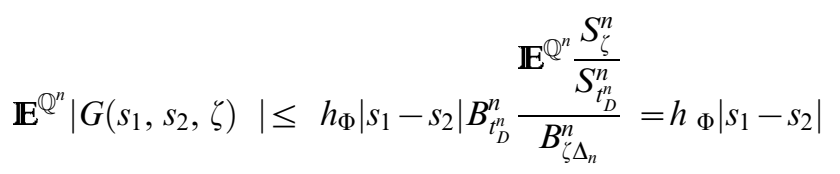

so since for all $s$ and $s_{1}$

$$
\begin{aligned}
f_{n}^{\mathrm{Am}}(s) & =B_{t_{D}^{n}}^{n} \sup _{\tau \in \mathcal{T}} \mathbb{E}_{\left\{\begin{array}{l}
\left.\mathbb{Q}^{n}, \ldots, T\right\} \\
\mathbb{Q}^{n}
\end{array}\right.}\left[\Phi\left(s \frac{S_{\tau}^{n}}{S_{t_{D}^{n}}^{n}}\right) / B_{\tau}^{n}\right] \\
& =\sup _{\tau \in \mathcal{T}} \mathbb{E}_{\left\{{ }^{n}, \ldots, T\right\}}^{\mathbb{Q}^{n}}\left[G\left(s, s_{1}, \tau\right)+B_{t_{D}^{n}}^{n} \Phi\left(s_{1} \frac{S_{\tau}^{n}}{S_{t_{D}^{n}}^{n}}\right) / B_{\tau}^{n}\right]
\end{aligned}
$$

we have

$$
\begin{aligned}
& f_{n}^{\mathrm{Am}}(s) \leq B_{t_{D}^{n}}^{n} \sup _{\tau \in \mathcal{T}} \mathbb{S}_{\left\{\begin{array}{l}
\left.\mathbb{Q}_{D}^{n}, \ldots, T\right\} \\
\mathbb{Q}^{n}
\end{array}\right.}\left[\Phi\left(s_{1} \frac{S_{\tau}^{n}}{S_{t_{D}^{n}}^{n}}\right) / B_{\tau}^{n}\right] \\
& +\sup _{\tau \in \mathcal{T}} \mathbb{E}_{\left\{t^{n}, \ldots, T\right\}}^{\mathbb{Q}^{n}}\left[\left|G\left(s, s_{1}, \tau\right)\right|\right] \\
& f_{n}^{\mathrm{Am}}(s) \geq B_{t_{D}^{n}}^{n} \sup _{\tau \in \mathcal{T}} \mathbb{E}_{\left\{l_{D}^{n}, \ldots, T\right\}} \mathbb{E}^{\mathbb{Q}^{n}}\left[\Phi\left(s_{1} \frac{S_{\tau}^{n}}{S_{t_{D}^{n}}^{n}}\right) / B_{\tau}^{n}\right] \\
& -\sup _{\tau \in \mathcal{T}} \mathbb{E}_{\left\{t_{D}^{n}, \ldots, T\right\}} \mathbb{E}^{\mathbb{Q}^{n}}\left[\left|G\left(s, s_{1}, \tau\right)\right|\right]
\end{aligned}
$$

so

$$
\left|f_{n}^{\mathrm{Am}}(s)-f_{n}^{\mathrm{Am}}\left(s_{1}\right)\right| \leq h_{\Phi}\left|s-s_{1}\right|
$$

which proves the result.

We can now prove our main result of the section.

Theorem 1. Under the assumptions (A1),(A2) and (A3), we have that for all $s \in \mathbb{R}^{+}$

$$
\begin{aligned}
& \lim _{n \rightarrow \infty} V_{n}^{\mathrm{Eur}}(s)=V^{\mathrm{Eur}}(s) \\
& \lim _{n \rightarrow \infty} V_{n}^{\operatorname{Am}}(s)=V^{\operatorname{Am}}(s)
\end{aligned}
$$

Proof. We will prove the American case; the European case is simpler and can be proven analogously. We have since $B_{0}^{n}=B_{0}$ 


$$
\begin{aligned}
& \frac{1}{B_{0}}\left(V_{n}^{\mathrm{Am}}(s)-V^{\mathrm{Am}}(s)\right) \\
& =\sup _{\tau \in \mathcal{T}} \mathbb{E}_{\left\{0, \Delta_{n}, \ldots, t_{D}^{n}\right\}}^{\mathbb{Q}^{n}}\left[\frac{\Phi\left(S_{\tau}\right)}{B_{\tau}} 1_{\left\{\tau<t_{D}\right\}}+1_{\left\{\tau=t_{D}\right\}} \frac{\mathcal{B}_{f_{n}^{\mathrm{Am}}}^{n}\left(S_{t_{D}^{n}-}^{n}-D\left(S_{t_{D}^{n}-}^{n}\right)\right)}{B_{t_{D}^{n}}^{n}} \mid S_{0}=s\right] \\
& \quad-\sup _{\tau \in \mathcal{T}_{\left[0, t_{D}\right]}} \mathbb{E}^{\mathbb{Q}}\left[\frac{\Phi\left(S_{\tau}\right)}{B_{\tau}} 1_{\left\{\tau<t_{D}\right\}}+1_{\left\{\tau=t_{D}\right\}} \frac{f^{\mathrm{Am}}\left(S_{t_{D}-}-D\left(S_{t_{D}-}\right)\right)}{B_{t_{D}}} \mid S_{0}=s\right] \\
& =E_{\text {approx }}^{n}(s)+E_{\mathrm{disc}}^{n}(s)
\end{aligned}
$$

where

$$
\begin{aligned}
& E_{\text {approx }}^{n}(s)=\sup _{\tau \in \mathcal{T}} \mathbb{E}_{\left\{0, \Delta_{n}, . ., t_{D}^{n}\right\}} \mathbb{E}^{\mathbb{Q}^{n}}\left[\frac{\Phi\left(S_{\tau}\right)}{B_{\tau}} 1_{\left\{\tau<t_{D}^{n}\right\}}+1_{\left\{\tau=t_{D}^{n}\right\}} \frac{\mathcal{B}_{f_{n}^{\text {Am }}}^{n}\left(S_{t_{D}^{n}-}^{n}-D\left(S_{t_{D}^{n}-}^{n}\right)\right)}{B_{t_{D}^{n}}} \mid S_{0}=s\right] \\
& -\sup _{\tau \in \mathcal{T}} \mathbb{E}_{\left\{0, \Delta_{n}, \ldots, t_{D}^{n}\right\}}^{\mathbb{Q}^{n}}\left[\frac{\Phi\left(S_{\tau}\right)}{B_{\tau}} 1_{\left\{\tau<t_{D}^{n}\right\}}+1_{\left\{\tau=t_{D}^{n}\right\}} \frac{f^{\mathrm{Am}}\left(S_{t_{D}^{n}-}^{n}-D\left(S_{t_{D}^{n}-}^{n}\right)\right)}{B_{t_{D}^{n}}} \mid S_{0}=s\right] \\
& E_{\text {disc }}^{n}(s)=\sup _{\tau \in \mathcal{T}} \mathbb{E}_{\left\{0, \Delta_{n}, \ldots, t_{D}^{n}\right\}} \mathbb{E}^{\mathbb{Q}^{n}}\left[\frac{\Phi\left(S_{\tau}\right)}{B_{\tau}} 1_{\left\{\tau<t_{D}^{n}\right\}}+1_{\left\{\tau=t_{D}^{n}\right\}} \frac{f^{\mathrm{Am}\left(S_{t_{D}^{n}-}^{n}-D\left(S_{t_{D}^{n}-}^{n}\right)\right)}}{B_{t_{D}^{n}}} \mid S_{0}=s\right] \\
& -\sup _{\tau \in \mathcal{T}_{\left[0, t_{D}\right]}} \mathbb{E}^{\mathbb{Q}}\left[\frac{\Phi\left(S_{\tau}\right)}{B_{\tau}} 1_{\left\{\tau<t_{D}\right\}}+1_{\left\{\tau=t_{D}\right\}} \frac{f^{\mathrm{Am}}\left(S_{t_{D}-}-D\left(S_{t_{D}-}\right)\right)}{B_{t_{D}}} \mid S_{0}=s\right]
\end{aligned}
$$

Now for all $s>0, E_{\text {disc }}^{\mathrm{n}}(s)$ converges to zero due to assumption (A3), since the requirement that all European and American option values converge for the case without dividends, allows us to apply the results of Amin and Khanna (1994), which show that we even have convergence for payoff functions which are time-varying (as they are in the expression above), since the uniform integrability condition on a timevarying payoff process $\Phi\left(S_{t}, t\right)$ which is needed for their result to hold true is satisfied because of our condition (A2). Hence, we just need to consider $E_{\text {approx }}^{n}(s)$.

Since both $f^{\mathrm{Am}}$ and $f_{n}^{\mathrm{Am}}$ are uniformly Lipschitz by Lemma 3 and since this property is inherited by linear interpolation, we can choose a $\widetilde{S}>0$ and $G>0$ with

$$
s>\widetilde{S} \Rightarrow\left|B_{f_{n}^{\mathrm{Am}}}^{n}(s-D(s))\right|+\left|f^{\mathrm{Am}}(s-D(s))\right|<G|s-D(s)| \leq G|s|
$$

for all $n$, where we used the fact that $s-D(s)$ was assumed to be positive. We can then, due to our condition (A1), choose for every $\varepsilon>0$ an $\bar{S}_{\varepsilon}>\widetilde{S}$ so large that 


$$
\left(\forall n \in \mathbb{N}^{+}\right) \mathbb{E}^{\mathbb{Q}^{n}}\left[S_{t_{D-}^{n}} 1_{\left\{S_{t_{D-}}^{n}>\bar{S}_{\varepsilon}\right\}} \mid S_{0}=s\right]<\frac{\varepsilon}{2 G} e^{-r T}
$$

Write

$$
E_{\text {approx }}^{n}(s)=\sup _{\tau \in \mathcal{T}} \mathbb{E}_{\left\{0, \Delta_{n}, \ldots, t_{D}^{n}\right\}} \mathbb{E}^{\mathbb{Q}^{n}}\left[\mathcal{E}_{1}^{n}(\tau) \mid S_{0}=s\right]-\sup _{\tau \in \mathcal{T}} \sup _{\left\{0, \Delta_{n}, \ldots, t_{D}^{n}\right\}} \mathbb{E}^{\mathbb{Q}^{n}}\left[\mathcal{E}_{2}^{n}(\tau) \mid S_{0}=s\right]
$$

where

$$
\begin{aligned}
& \mathcal{E}_{1}^{n}(\tau)=\frac{\Phi\left(S_{\tau}\right)}{B_{\tau}} 1_{\left\{\tau<t_{D}^{n}\right\}}+1_{\left\{\tau=t_{D}^{n}\right\}} \frac{\mathcal{B}_{f_{n}^{\mathrm{Am}}}^{n}\left(S_{t_{D}^{n}-}^{n}-D\left(S_{t_{D}^{n}-}^{n}\right)\right)}{B_{t_{D}^{n}}} \\
& \mathcal{E}_{2}^{n}(\tau)=\frac{\Phi\left(S_{\tau}\right)}{B_{\tau}} 1_{\left\{\tau<t_{D}\right\}}+1_{\left\{\tau=t_{D}\right\}} \frac{f^{\mathrm{Am}}\left(S_{t_{D}-}-D\left(S_{t_{D}-}\right)\right)}{B_{t_{D}}}
\end{aligned}
$$

and by (5),

$$
\begin{aligned}
& \mathcal{E}_{1}^{n}(\tau) \leq \frac{\Phi\left(S_{\tau}\right)}{B_{\tau}} 1_{\left\{\tau<t_{D}^{n}\right\}}+1_{\left\{S_{t_{D-}^{n}} \leq \tilde{S}_{\varepsilon}\right\}} 1_{\left\{\tau=t_{D}^{n}\right\}} \frac{\mathcal{B}_{f_{n}^{\mathrm{Am}}}^{n}\left(S_{t_{D}^{n}-}^{n}-D\left(S_{t_{D}^{n}-}^{n}\right)\right)}{B_{t_{D}^{n}}}+G\left|S_{t_{D-}^{n}}\right| 1_{\left\{S_{t_{D-}^{n}}>\tilde{S}_{\varepsilon}\right\}} \\
& \mathcal{E}_{2}^{n}(\tau) \geq \frac{\Phi\left(S_{\tau}\right)}{B_{\tau}} 1_{\left\{\tau<t_{D}^{n}\right\}}+1_{\left\{S_{t_{D-}^{n}} \leq \tilde{S}_{\varepsilon}\right\}} 1_{\left\{\tau=t_{D}^{n}\right\}} \frac{f^{\mathrm{Am}\left(S_{t_{D}^{n}-}^{n}-D\left(S_{t_{D}^{n}-}^{n}\right)\right)}}{B_{t_{D}^{n}}}-G\left|S_{t_{D-}^{n}}\right| 1_{\left\{S_{t_{D-}^{n}}>\tilde{S}_{\varepsilon}\right\}}
\end{aligned}
$$

and together with (6) this shows that

$$
\begin{aligned}
& \left|E_{\text {approx }}^{n}(s)\right| \leq \varepsilon+ \\
& \mid \sup _{\tau \in \mathcal{T}} \mathbb{E}_{\left\{0, \Delta_{n}, \ldots, t_{D}^{n}\right\}}^{\mathbb{Q}^{n}}\left[\frac{\Phi\left(S_{\tau}\right)}{B_{\tau}} 1_{\left\{\tau<t_{D}^{n}\right\}}+1_{\left\{S_{t_{D-}^{n}} \leq \tilde{S}_{\varepsilon}\right\}^{1}}{ }_{\left\{\tau=t_{D}^{n}\right\}} \frac{\mathcal{B}_{f_{n}^{\mathrm{Am}}}^{n}\left(S_{t_{D}^{n}-}^{n}-D\left(S_{t_{D}^{n}-}^{n}\right)\right)}{B_{t_{D}^{n}}} \mid S_{0}=s\right] \\
& -\sup _{\tau \in \mathcal{T}} \mathbb{E}_{\left\{0, \Delta_{n}, \ldots, t_{D}^{n}\right\}}^{\mathbb{Q}^{n}}\left[\frac{\Phi\left(S_{\tau}\right)}{B_{\tau}} 1_{\left\{\tau<t_{D}^{n}\right\}}+1_{\left\{S_{t_{D-}^{n}} \leq \tilde{S}_{\varepsilon}\right\}} 1_{\left\{\tau=t_{D}^{n}\right\}} \frac{f^{\mathrm{Am}}\left(S_{t_{D}^{n}-}^{n}-D\left(S_{t_{D}^{n}-}^{n}\right)\right)}{B_{t_{D}^{n}}} \mid S_{0}=s\right] \mid
\end{aligned}
$$

We are clearly done if we can prove that

$$
\lim _{n \rightarrow \infty} \sup _{0 \leq s \leq \tilde{S}_{\varepsilon}}\left|\mathcal{B}_{f_{n}^{\text {Am }}}^{n}(s)-f^{\mathrm{Am}}(s)\right|=0
$$

because if

$$
(\forall \delta>0)\left(\exists R_{\delta} \in \mathbb{N}^{+}\right)\left(\forall n>R_{\delta}\right) \sup _{0 \leq s \leq \tilde{S}_{\varepsilon}}\left|\mathcal{B}_{f_{n}^{\mathrm{Am}}}^{n}(s)-f^{\mathrm{Am}}(s)\right|<\delta
$$


then we we have that for $n>R_{\delta}$

$$
\begin{aligned}
& \left|E_{\text {approx }}^{n}\right| \leq \varepsilon+ \\
& \mid \sup _{\tau \in \mathcal{T}} \mathbb{E}_{\left\{0, \Delta_{n}, \ldots, t_{D}^{n}\right\}}^{\mathbb{Q}^{n}}\left[\frac{\Phi\left(S_{\tau}\right)}{B_{\tau}} 1_{\left\{\tau<t_{D}^{n}\right\}}+{ }_{\left\{S_{t_{D-}^{n}} \leq \tilde{S}_{\varepsilon}\right\}} 1_{\left\{\tau=t_{D}^{n}\right\}}\left(\delta+\frac{f^{\mathrm{Am}}\left(S_{t_{D}^{n}-}^{n}-D\left(S_{t_{D}^{n}-}^{n}\right)\right)}{B_{t_{D}^{n}}}\right) \mid S_{0}=S\right] \\
& -\sup _{\tau \in \mathcal{T}} \mathbb{E}_{\left\{0, \Delta_{n}, \ldots, t_{D}^{n}\right\}}^{\mathbb{Q}^{n}}\left[\frac{\Phi\left(S_{\tau}\right)}{B_{\tau}} 1_{\left\{\tau<t_{D}^{n}\right\}}+1_{\left\{S_{t_{D-}^{n}} \leq \tilde{S}_{\varepsilon}\right\}} 1_{\left\{\tau=t_{D}^{n}\right\}} \frac{f^{\mathrm{Am}\left(S_{t_{D}^{n}-}^{n}-D\left(S_{t_{D}^{n}-}^{n}\right)\right)}}{B_{t_{D}^{n}}} \mid S_{0}=S\right] \mid \\
& \leq \delta+\varepsilon
\end{aligned}
$$

for $\delta$ and $\varepsilon$ arbitrarily small. To prove (7), take $n>N_{\varepsilon}$ so large that $\max \left(A^{n}\right)>\widetilde{S}_{\varepsilon}$ (this is possible since $\max \left(A^{n}\right) \rightarrow \infty$ for $n \rightarrow \infty$ because otherwise (A3) will clearly not hold for certain choices of $\Phi)$. We set

$$
a_{n}(s)=\arg \min _{x \in A^{n}}|x-s|
$$

and write

$$
\begin{aligned}
\left|\mathcal{B}_{f_{n}^{\text {Am }}}^{n}(s)-f^{\mathrm{Am}}(s)\right| \leq & \left|\mathcal{B}_{f_{n}^{\text {Am }}}^{n}(s)-\mathcal{B}_{f_{n}^{\text {Am }}}^{n}\left(a_{n}(s)\right)\right|+\left|\mathcal{B}_{f_{n}^{\text {Am }}}^{n}\left(a_{n}(s)\right)-f_{n}^{\mathrm{Am}}\left(a_{n}(s)\right)\right| \\
& +\left|f_{n}^{\mathrm{Am}}\left(a_{n}(s)\right)-f^{\mathrm{Am}}\left(a_{n}(s)\right)\right|+\left|f^{\mathrm{Am}}\left(a_{n}(s)\right)-f^{\mathrm{Am}}(s)\right|
\end{aligned}
$$

the second term is zero by definition and we can bound the first and last term using the uniform Lipschitz properties that we derived before equation (6) for both $\mathcal{B}_{f_{n}^{\text {Am }}}^{\mathrm{n}}(s)$ and $f^{\mathrm{Am}}$ (say the values of the Lipschitz constants of the functions do not exceed $H>0$ ), so

$$
\left|\mathcal{B}_{f_{n}^{\mathrm{Am}}}^{n}(s)-f^{\mathrm{Am}}(s)\right| \leq 2 H \cdot \operatorname{mesh}\left(A^{n}\right)+\left|f_{n}^{\mathrm{Am}}\left(a_{n}(s)\right)-f^{\mathrm{Am}}\left(a_{n}(s)\right)\right|
$$

where $\operatorname{mesh}\left(A^{n}\right)=\max \left\{A_{1}^{n}-0, \max _{k=1 . . n-1}\left(A_{k+1}^{n}-A_{k}^{n}\right)\right\}$ converges to zero for $n \rightarrow \infty$, since otherwise (A3) would clearly not hold. We now write

$$
\begin{aligned}
& \sup _{0 \leq s \leq \tilde{S}_{\varepsilon}}\left|\mathcal{B}_{f_{n}^{\mathrm{Am}}}^{n}(s)-f^{\mathrm{Am}}(s)\right| \\
& \leq 2 H \cdot \operatorname{mesh}\left(A^{n}\right)+\sup _{0 \leq s \leq \tilde{S}_{\varepsilon}}\left|f_{n}^{\mathrm{Am}}\left(a_{n}(s)\right)-f^{\mathrm{Am}}\left(a_{n}(s)\right)\right|
\end{aligned}
$$

but we assumed that the convergence of $f_{n}^{\mathrm{Am}}$ to $f^{\mathrm{Am}}$ for American options without dividend is uniform on closed bounded subsets of $\mathbb{R}^{+}$and this implies that

$$
\lim _{n \rightarrow \infty} \sup _{0 \leq s \leq \widetilde{S}_{\varepsilon}}\left|\mathcal{B}_{f_{n}^{\mathrm{Am}}}^{n}(s)-f^{\mathrm{Am}}(s)\right|=0 .
$$

This proves (7) and hence the result. 


\section{Remarks}

- In practical cases, where one can obviously use only a finite number of time steps, it is important to make sure that the approximation step is accurate enough, i.e. that there are enough nodes in the tree at the first dividend date.

- Note that for convex pricing functions, the linear interpolation step always overestimates the value that it tries to approximate.

- One could use a cubic spline approximation instead of a linear approximation. To prove convergence we needed a Lipschitz condition for the tail in Equation 6 and in the error bound in Equation 8. A cubic spline approximation will not automatically inherit the Lipschitz property of the function it approximates, so the proof given does not go through directly. However, if one applies a cubic spline method which inherits monotonicity from the function it approximates, then the proof can easily be shown to go through if the price functions $f^{n}$ and f are monotone (which is clearly the case for standard Call and Put contracts). Such spline methods exist (Kuijt and VanDamme, 1999).

- We have only given the proof for the case of one dividend, but the extension to more dividends is obvious. Note however, that one has to check that the conditions (A1), (A2), and (A3) remain valid for every single step, which may be complicated for exotic payoff functions, since little is known in general about the rates of convergence for such cases.

\section{Numerical Results}

To illustrate results, the proposed method and other available methods were tested on a range of European and American options. A standard binomial tree and linear interpolation were used in all cases described below.

In a first experiment, we price an American call with time to maturity 1 year and a single dividend of value 7.0 at time $t_{1}$, where $t_{1}$ was taken to be $0.1,0.5$ and 0.9 in different experiments. As is well known, the exact value of this American option can be calculated using a single integral. The volatility and interest rate were taken to be $\sigma=30 \%$ and $r=5 \%$ respectively, and the current stock price was $S=100$. We took strike prices equal to 70,100 and 130 . Note that quite a long time to maturity and high dividends were taken to make differences between the pricing methods more transparent.

Table 1 shows the exact value, the results of our algorithm (VN) for 250, 500 and 1000 steps in the binomial tree respectively, and the results for the two other available methods to deal with American options with a single dividend, the RollGeske-Whaley method (RGW) and the Black approximation (see Roll, 1977; Whaley, 1981; Geske, 1979; Black, 1975) for more information on these methods). We also give the results (VNRE) of Richardson extrapolation on our method (where the maximal number of steps used was 64000) to show that we have convergence. Note that the results are very satisfactory for a more moderate number of steps, while the two other models may significantly misprice the options.

In the second experiment, we considered a European call with a time to maturity of 7 years and cash dividends equal to $6.0,6.5,7.0,7.5,8.0,8.0,8.0$, in consecutive 
Table 1. American call, $\sigma=30 \%, r=5 \%, S=100$

\begin{tabular}{|c|c|c|c|c|c|c|c|c|}
\hline$t_{1}$ & $K$ & Exact & VNRE & VN1000 & VN500 & VN250 & RGW & Black \\
\hline \multirow[t]{3}{*}{0.1} & 70 & 30.38 & 30.38 & 30.38 & 30.37 & 30.35 & 30.37 & 30.35 \\
\hline & 100 & 10.29 & 10.29 & 10.29 & 10.29 & 10.28 & 10.20 & 10.20 \\
\hline & 130 & 3.00 & 3.00 & 3.00 & 3.00 & 2.99 & 2.93 & 2.93 \\
\hline \multirow[t]{3}{*}{0.5} & 70 & 32.13 & 32.13 & 32.12 & 32.11 & 32.10 & 32.00 & 31.98 \\
\hline & 100 & 11.33 & 11.33 & 11.32 & 11.31 & 11.30 & 10.86 & 10.28 \\
\hline & 130 & 3.28 & 3.28 & 3.28 & 3.28 & 3.28 & 2.98 & 2.96 \\
\hline \multirow[t]{3}{*}{0.9} & 70 & 33.92 & 33.92 & 33.91 & 33.90 & 33.88 & 33.66 & 33.91 \\
\hline & 100 & 13.49 & 13.49 & 13.48 & 13.47 & 13.43 & 12.75 & 13.40 \\
\hline & 130 & 4.17 & 4.17 & 4.16 & 4.15 & 4.14 & 3.62 & 4.04 \\
\hline \multirow[t]{3}{*}{0.1} & 70 & & $0.00 \%$ & $-0.03 \%$ & $-0.06 \%$ & $-0.11 \%$ & $-0.04 \%$ & $-0.12 \%$ \\
\hline & 100 & & $0.00 \%$ & $0.00 \%$ & $0.00 \%$ & $-0.05 \%$ & $-0.84 \%$ & $-0.84 \%$ \\
\hline & 130 & & $0.00 \%$ & $0.04 \%$ & $0.14 \%$ & $-0.04 \%$ & $-2.07 \%$ & $-2.07 \%$ \\
\hline \multirow[t]{3}{*}{0.5} & 70 & & $0.00 \%$ & $-0.03 \%$ & $-0.06 \%$ & $-0.10 \%$ & $-0.40 \%$ & $-0.48 \%$ \\
\hline & 100 & & $0.00 \%$ & $-0.06 \%$ & $-0.12 \%$ & $-0.27 \%$ & $-4.13 \%$ & $-9.26 \%$ \\
\hline & 130 & & $0.00 \%$ & $0.01 \%$ & $0.05 \%$ & $-0.01 \%$ & $-9.03 \%$ & $-9.66 \%$ \\
\hline \multirow[t]{3}{*}{0.9} & 70 & & $0.00 \%$ & $-0.03 \%$ & $-0.06 \%$ & $-0.10 \%$ & $-0.76 \%$ & $-0.03 \%$ \\
\hline & 100 & & $0.00 \%$ & $-0.11 \%$ & $-0.17 \%$ & $-0.46 \%$ & $-5.49 \%$ & $-0.64 \%$ \\
\hline & 130 & & $0.00 \%$ & $-0.17 \%$ & $-0.36 \%$ & $-0.63 \%$ & $-13.28 \%$ & $-3.09 \%$ \\
\hline
\end{tabular}

years. The time between dividend dates was taken to be one year, and the time of the first dividend was again varied: we present results for $t_{1}=0.1, t_{1}=0.5$ and $t_{1}=0.9$ with $\sigma=25 \%, r=6 \%$ and $S=100$.

The results are shown in Table 2 for our methods with 250, 500 and 1000 time steps, and alternative methods formulated by Haug, Haug and Lewis (2003, HHL), Bos, Gairat and Shepelva (2003, BGS), Bos and Vandermark (2002, BvdM) and Beneder and Vorst $(2002$, BV). The exact details of their methods can be found in the corresponding papers in the references.

As we can see, the last three methods show substantial differences when compared to our method. The approximation method of Haug, Haug and Lewis (who replace a multiple integration by a succession of single integrations over Black-Scholes-like approximating functions) performs extremely well. In fact it outperforms the other existing methods in all cases and outperforms the proposed method when only 250 steps are used. When our method uses more steps, it gives results closer to the correct value (as expected, since we actually proved convergence). However, it should be noted that the last three methods in the table are obviously quicker to calculate since they are all based on the Black-Scholes formula for European options in which an adjusted volatility is inserted, so there is a trade-off in accuracy and time involved here.

In the last experiment we took the same options as before, but this time looked at the American call option. None of the other methods mentioned above can deal with American options with multiple dividends and no exact formula exists. We again note that finite difference methods can also be used to price American options in the presence of multiple cash dividends, but to be sure that there is convergence to the correct value, a detailed analysis similar to ours would be needed. 
Table 2. European call, $\sigma=25 \%, r=6 \%, S=100$

\begin{tabular}{rrrrrrrrrc}
\hline$t_{1}$ & $K$ & VNRE & VN1000 & VN500 & VN250 & HHL & BGS & BvdM & BV \\
\hline 0.1 & 70 & 24.90 & 24.92 & 24.98 & 25.24 & 25.05 & 24.71 & 24.74 & 23.43 \\
& 100 & 17.43 & 17.46 & 17.51 & 17.74 & 17.50 & 17.42 & 17.08 & 16.41 \\
& 130 & 12.40 & 12.43 & 12.47 & 12.69 & 12.40 & 12.50 & 11.94 & 11.83 \\
0.5 & 70 & 26.08 & 26.10 & 26.10 & 26.14 & 26.20 & 25.87 & 25.94 & 24.58 \\
& 100 & 18.48 & 18.50 & 18.51 & 18.56 & 18.51 & 18.45 & 18.15 & 17.51 \\
& 130 & 13.29 & 13.31 & 13.33 & 13.40 & 13.24 & 13.38 & 12.84 & 12.83 \\
0.9 & 70 & 27.21 & 27.23 & 27.23 & 27.28 & 27.30 & 26.99 & 27.10 & 25.67 \\
& 100 & 19.48 & 19.50 & 19.52 & 19.55 & 19.48 & 19.43 & 19.19 & 18.54 \\
& 130 & 14.13 & 14.16 & 14.17 & 14.25 & 14.06 & 14.21 & 13.73 & 13.77 \\
\hline 0.1 & 70 & & $0.10 \%$ & $0.32 \%$ & $1.39 \%$ & $0.62 \%$ & $-0.74 \%$ & $-0.64 \%$ & $-5.89 \%$ \\
& 100 & & $0.16 \%$ & $0.43 \%$ & $1.73 \%$ & $0.39 \%$ & $-0.11 \%$ & $-2.01 \%$ & $-5.86 \%$ \\
& 130 & & $0.27 \%$ & $0.59 \%$ & $2.30 \%$ & $-0.03 \%$ & $0.81 \%$ & $-3.69 \%$ & $-4.56 \%$ \\
0.5 & 70 & & $0.07 \%$ & $0.08 \%$ & $0.24 \%$ & $0.44 \%$ & $-0.80 \%$ & $-0.54 \%$ & $-5.75 \%$ \\
& 100 & & $0.12 \%$ & $0.17 \%$ & $0.41 \%$ & $0.15 \%$ & $-0.19 \%$ & $-1.78 \%$ & $-5.28 \%$ \\
& 130 & & $0.22 \%$ & $0.30 \%$ & $0.88 \%$ & $-0.32 \%$ & $0.71 \%$ & $-3.32 \%$ & $-3.45 \%$ \\
0.9 & 70 & & $0.07 \%$ & $0.08 \%$ & $0.23 \%$ & $0.31 \%$ & $-0.83 \%$ & $-0.41 \%$ & $-5.68 \%$ \\
& 100 & & $0.10 \%$ & $0.17 \%$ & $0.37 \%$ & $-0.01 \%$ & $-0.27 \%$ & $-1.50 \%$ & $-4.85 \%$ \\
& 130 & & $0.21 \%$ & $0.28 \%$ & $0.87 \%$ & $-0.47 \%$ & $0.57 \%$ & $-2.86 \%$ & $-2.58 \%$ \\
\hline
\end{tabular}

We therefore again tried to find its value by applying the proposed method, using a very large number of steps (64000) and Richardson extrapolation, and compared this value to the same method using a more modest numbers of steps and to the results of the Longstaff and Schwartz (2001) method of Monte Carlo simulation for American options. The results can be found in Table 3. We see that for 1000 steps we already have very satisfactory results but for a smaller number of steps we may run into problems when the first dividend comes early, since the approximation of the option values at that point is then based on too few interpolation points. As mentioned in the preceding section, more time steps are needed to obtain good results in this case.

Notice that the results of the Monte Carlo method of Longstaff and Schwarz are close to ours. The Monte Carlo estimates are based on 2000000 antithetic samples and three basis functions in the Longstaff-Schwartz least squares approximation. The maximal absolute error of $1 \%$ we observe for the Monte Carlo prices is not exceptional: we also ran the Monte Carlo simulation for the setup of Table 1 (where we know the exact value of the option) and found a maximal absolute error of $2 \%$ there.

Finally, in Table 4 we took the same setup as in the previous experiment, but we now took all cash dividends equal to 3 , and we removed the first and last dividend dates. This ensures that for all cases considered here we have that

$$
D<K\left(1-e^{-r\left(t_{D}^{i+1}-t_{D}^{i}\right)}\right)
$$

for $i=0 \ldots 6$, where $\left\{t_{D}^{1}, t_{D}^{2}, \ldots, t_{D}^{6}\right\}$ are the dividend dates and $t_{D}^{0}=0$ and $t_{D}^{7}=T$. This condition guarantees that it is never optimal to exercise the American option and we 
Table 3. American call, $\sigma=25 \%, r=6 \%, S=100$

\begin{tabular}{rrrrrrr}
\hline$t_{1}$ & $K$ & VNRE & VN1000 & VN500 & VN250 & LonSch \\
\hline 0.1 & 70 & 31.14 & 31.06 & 31.07 & 31.25 & 30.91 \\
& 100 & 18.32 & 18.32 & 18.35 & 18.54 & 18.17 \\
& 130 & 12.48 & 12.51 & 12.54 & 12.73 & 12.57 \\
0.5 & 70 & 33.47 & 33.40 & 33.37 & 33.27 & 33.30 \\
& 100 & 20.04 & 20.04 & 20.02 & 20.00 & 20.07 \\
& 130 & 13.75 & 13.76 & 13.75 & 13.78 & 13.80 \\
0.9 & 70 & 35.52 & 35.48 & 35.42 & 35.31 & 35.28 \\
& 100 & 21.86 & 21.85 & 21.82 & 21.78 & 21.65 \\
& 130 & 15.21 & 15.21 & 15.19 & 15.21 & 15.09 \\
\hline 0.1 & 70 & & $-0.24 \%$ & $-0.22 \%$ & $0.36 \%$ & $-0.69 \%$ \\
& 100 & & $0.03 \%$ & $0.16 \%$ & $1.18 \%$ & $-0.80 \%$ \\
& 130 & & $0.20 \%$ & $0.45 \%$ & $2.02 \%$ & $0.71 \%$ \\
0.5 & 70 & & $-0.19 \%$ & $-0.29 \%$ & $-0.58 \%$ & $-0.45 \%$ \\
& 100 & & $-0.03 \%$ & $-0.14 \%$ & $-0.25 \%$ & $0.15 \%$ \\
& 130 & & $0.10 \%$ & $0.00 \%$ & $0.26 \%$ & $0.36 \%$ \\
0.9 & 70 & & $-0.13 \%$ & $-0.30 \%$ & $-0.60 \%$ & $-0.66 \%$ \\
& 100 & & $-0.07 \%$ & $-0.18 \%$ & $-0.35 \%$ & $-0.96 \%$ \\
& 130 & & $0.01 \%$ & $-0.11 \%$ & $-0.02 \%$ & $-0.82 \%$ \\
\hline
\end{tabular}

should therefore find exactly the same prices when we use the European or the American version of our algorithm. We see in Table 4 that the prices are indeed exactly the same. We also find excellent agreement with Monte Carlo estimates,

Table 4: American Call, $\sigma=25 \%, r=6 \%, S=100$

\begin{tabular}{lrccc}
\hline$t_{1}$ & $K$ & VN5000 Am & VN5000 Eur & Monte Carlo \\
\hline 0.1 & 70 & 45.873 & 45.873 & 45.878 \\
& 100 & 33.724 & 33.724 & 33.722 \\
0.5 & 130 & 24.868 & 24.868 & 24.857 \\
& 70 & 46.238 & 46.238 & 46.232 \\
& 100 & 34.089 & 34.089 & 34.085 \\
0.9 & 130 & 25.206 & 25.206 & 25.195 \\
& 70 & 46.594 & 46.594 & 46.591 \\
& 100 & 34.443 & 34.443 & 34.440 \\
0.1 & 130 & 25.532 & 25.532 & 25.533 \\
& 70 & & $0.000 \%$ & $0.010 \%$ \\
0.5 & 100 & & $0.000 \%$ & $-0.006 \%$ \\
& 130 & & $0.000 \%$ & $-0.045 \%$ \\
& 70 & & $0.000 \%$ & $-0.013 \%$ \\
0.9 & 100 & & $0.000 \%$ & $-0.012 \%$ \\
& 130 & & $0.000 \%$ & $-0.045 \%$ \\
& 70 & & $0.000 \%$ & $-0.007 \%$ \\
\hline
\end{tabular}


which were generated using 2000000 antithetic samples and with control variates consisting of European options with the same parameters but without dividends.

\section{Conclusions}

We have presented an efficient method to deal with cash dividends in equity option pricing methods, under the assumption that in between dividend dates the asset follows lognormal dynamics, and where the same dynamics are used to price all derivative products. The interpolation method used was shown to converge under an additional assumption of uniform convergence for approximation methods without dividends, since this allows one to use a conditioning argument over the dividend dates.

Our method does not give closed-form formulas for European options (such as the Beneder-Vorst, the Bos-VdMark and the Bos-Gairat-Shepeleva models) but it has the advantage that it can also be used for American options with multiple dividends. Since the interpolation step takes only a modest amount of time, the speed of the proposed method is roughly the same as that of the underlying binomial, trinomial or other schemes without dividends on which our method can be based. We therefore expect it to be useful for consistent pricing of derivatives on assets which involve cash dividends.

\section{Acknowledgements}

This research was partially funded by The Derivatives Technology Foundation. Their support is gratefully acknowledged.

\section{References}

Amin, K. and Khanna, A. (1994) Convergence of American option values from discrete- to continuoustime financial models, Mathematical Finance, 4, pp. 289-304.

Beneder, R. and Vorst, T. (2002) Options on dividend paying stocks, in: J. Yong (Ed.) Recent Developments in Mathematical finance (Shanghai, 2001) (River Edge, NJ: World Scientific Publishing).

Black, F. (1975) Fact and fantasy in the use of options, Financial Analysts Journal, pp. 36-72.

Black, F. and Scholes, M. (1973) The pricing of options and corporate liabilities, Journal of Political Economy, 81, pp. 637-654.

Bos, R. et al. (2003) Dealing with discrete dividends, Risk Magazine, 16, pp. 109-112.

Bos, R. and Vandermark, S. (2002) Finessing fixed dividends, Risk Magazine, 15, pp. 157-158.

Cox, J. C. et al. (1979) Option pricing: a simplified approach, Journal of Financial Economics, 7, pp. 229-263.

Frishling, V. (2002) A discrete question, Risk Magazine, 15, pp. 115-116.

Geske, R. (1979) A note on an analytical formula for unprotected american call options on stocks with known dividends, Journal of Financial Economics, 7, pp. 375-380.

Haug, E. G. et al. (2003) Back to basics: a new approach to the discrete dividend problem, Wilmott Magazine, pp. 37-47.

Kuijt, F. and VanDamme, R. (1999) Monotonicity preserving interpolatory subdivision schemes, Journal of Computational \& Applied Mathematics, 101, pp. 203-229.

Lamberton, D. (1998) Error estimates for the binomial approximation of american put options, Annals of Applied Probability, 8, pp. 206-233.

Leisen, D. P. (1998) Pricing the american put option: a detailed convergence analysis for binomial models, Journal Economics Dynamics and Control, 22, pp. 1419-1444. 
284 M. H. Vellekoop and J. W. Nieuwenhuis

Longstaff, F. A. and Schwartz, E. S. (2001) Valuing American options by simulation: a simple leastsquares method, Review of Financial Studies, 14, pp. 113-147.

Merton, R. C. (1973) Theory of rational option pricing, Bell Journal of Economics and Management Science, 4, pp. 141-183.

Roll, R. (1977) An analytical formula for unprotected american call options on stocks with known dividends, Journal of Financial Economics, 5, pp. 251-258.

Whaley, R. E. (1981) On the valuation of american call options on stocks with known dividends, Journal of Financial Economics, 9, pp. 207-211.

Wilmott, P. et al. (1993) Option Pricing: Mathematical Models and Computation (Oxford: Oxford Financial Press). 
Copyright of Applied Mathematical Finance is the property of Routledge and its content may not be copied or emailed to multiple sites or posted to a listserv without the copyright holder's express written permission. However, users may print, download, or email articles for individual use. 